\title{
Descrição da fêmea de Sphecozone tincta (Araneae, Linyphiidae) e novas ocorrências no Brasil ${ }^{1}$
}

\author{
Everton Nei Lopes Rodrigues ${ }^{2}$
}

1. Trabalho realizado no Museu de Ciências Naturais, Fundação Zoobotânica do Rio Grande do Sul.

2. Programa de Pós-Graduação em Biologia Animal, Departamento de Zoologia, Instituto de Biociências, Universidade Federal do Rio Grande do Sul, Av. Bento Gonçalves, 9500, Bloco IV, Prédio 43435, 91501-970 Porto Alegre, RS, Brasil. (enlrodrigues@yahoo.com.br)

\begin{abstract}
Description of the female of Sphecozone tincta (Araneae, Linyphiidae) and news records from Brazil. The female of Sphecozone tincta Millidge, 1991 is described and illustrated for the first time. The male also is illustrated. News records are given for Sphecozone cristata Millidge, 1991, S. rostrata Millidge, 1991 and S. rubescens O. P.-Cambridge, 1870.
\end{abstract}

KEYWORDS. Araneae, Linyphiidae, Sphecozone, Neotropical, taxonomy.

RESUMO. A fêmea de Sphecozone tincta Millidge, 1991 é descrita e ilustrada pela primeira vez. O macho também é ilustrado. Novos registros são fornecidos para Sphecozone cristata Millidge, 1991, S. rostrata Millidge, 1991 e S. rubescens O. P.-Cambridge, 1870.

PALAVRAS-CHAVE. Araneae, Linyphiidae, Sphecozone, Neotropical, taxonomia.

O gênero Sphecozone foi proposto por PICKARDCAmbridge (1870), designando a espécie-tipo Sphecozone rubescens O. P.-Cambridge, 1870. MILLIDGE (1985) citou o gênero como provavelmente endêmico para a América do Sul. Na atualidade, há registro de uma espécie para a América do Norte (MILLIDGE, 1993). Atualmente o gênero é composto por 26 espécies, sendo sete para o Brasil (Platnick, 2004). Millidge (1991), num extenso trabalho sobre aranhas da família Linyphiidae da América do Sul, descreveu vários gêneros e espécies, entre as quais Sphecozone tincta, com base em um macho coletado em Pelotas, Rio Grande do Sul, Brasil.

Durante coletas realizadas na Estação Experimental do Arroz, do Instituto Rio Grandense do Arroz, em Cachoeirinha, RS, foram coletados macho e fêmea de $S$. tincta. Descreve-se a fêmea pela primeira vez e são dadas ilustrações do macho. Sphecozone cristata Millidge, 1991, S. rostrata Millidge, 1991 e S. rubescens O. P.-Cambridge, 1870 são registradas pela primeira vez para a região Sul do Brasil, ampliando-se suas distribuições.

Os espécimes examinados estão depositados na Coleção de Aranhas do Museu de Ciências Naturais, Fundação Zoobotânica do Rio Grande do Sul, MCN (E. H. Buckup), Porto Alegre. O cálculo para a posição da tricobótria no metatarso da perna I $(\mathrm{TmI})$ foi realizado conforme Millidge (1980). Descrições seguiram Millidge $(1985,1991)$. Para o estudo da estrutura interna da genitália da fêmea, o epígino foi imerso em óleo de cravo. Medidas são dadas em milímetros (mm).

\section{Sphecozone tincta Millidge, 1991} (Figs. 1-11)

Sphecozene tincta Millidge, 1991:174; figs. 731, 732, 737,

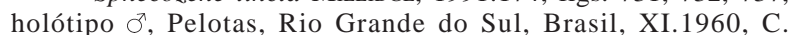
Biezanko col., depositado no American Museum of Natural History (AMNH) (não examinado); Platnick, 1993: 354.
Diagnose. O palpo do macho de S. tincta, semelhante ao de S. cristata Millidge, 1991 (Millidge, 1991, figs. 729, 730), difere pela acentuada dilatação ventral da tíbia e pelo percurso da porção distal do êmbolo direcionado para o ápice do címbio (figs. 1, 2). Fêmea com epígino similar ao de S. fuscipes Millidge, 1991 (Millidge, 1991, fig. 725); distingue-se pelo átrio com as bordas das aberturas de copulação semi-circulares e pela porção posterior divergente; na margem anterior do epígino, por duas depressões, uma de cada lado, maiores e mais próximas entre si (fig. 9).

Descrição. O macho foi descrito por MilLidge (1991), aqui suplementado por ilustrações do corpo em vistas ventral, dorsal e lateral (figs. 3-5) e palpo em vista ectal e mesal (figs. 1,2). Comprimento total 2,30. Carapaça, comprimento 1,07, largura 0,80, região cefálica 0,57. Clípeo, altura 0,30. Abdômen, comprimento 1,27, largura 0,80, altura 0,82. Pernas 1,4,2,3. Comprimento I/II/III/IV: fêmures $1,10 / 1,02 / 0,82 / 1,05$; patelas $0,27 / 0,25 / 0,20 / 0,25$; tíbias $0,97 /$ $0,92 / 0,70 / 0,92$; metatarsos $0,85 / 0,77 / 0,62 / 0,87$; tarsos $0,50 /$ $0,47 / 0,42 / 0,47$. Total 3,69/3,43/2,76/3,56. Cálculo para a posição da tricobótria no metatarso da perna I (TmI) 0,40. Metatarsos II, III e IV também possuem tricobótrias. Carapaça castanho-avermelhada; área ocular escurecida, mais larga $(0,40)$ do que longa $(0,16)$. Quadrângulo ocular médio: largura anterior 0,11 , comprimento 0,16 , largura posterior 0,18. Esterno castanho-avermelhado com tonalidade mais escura que a carapaça, densamente piloso. Quelíceras e enditos castanho-escuros. Pernas castanho-claras com patelas e tíbias escurecidas, coxas e trocanteres mais claros em relação aos demais artículos. Abdômen castanho-alaranjado e piloso, área das fiandeiras mais escura.

Fêmea (MCN 38089). Comprimento total 2,32. Carapaça, comprimento 1,12 , largura 0,87 , região cefálica 0,62. Clípeo, altura 0,22. Abdômen, comprimento 1,22, 

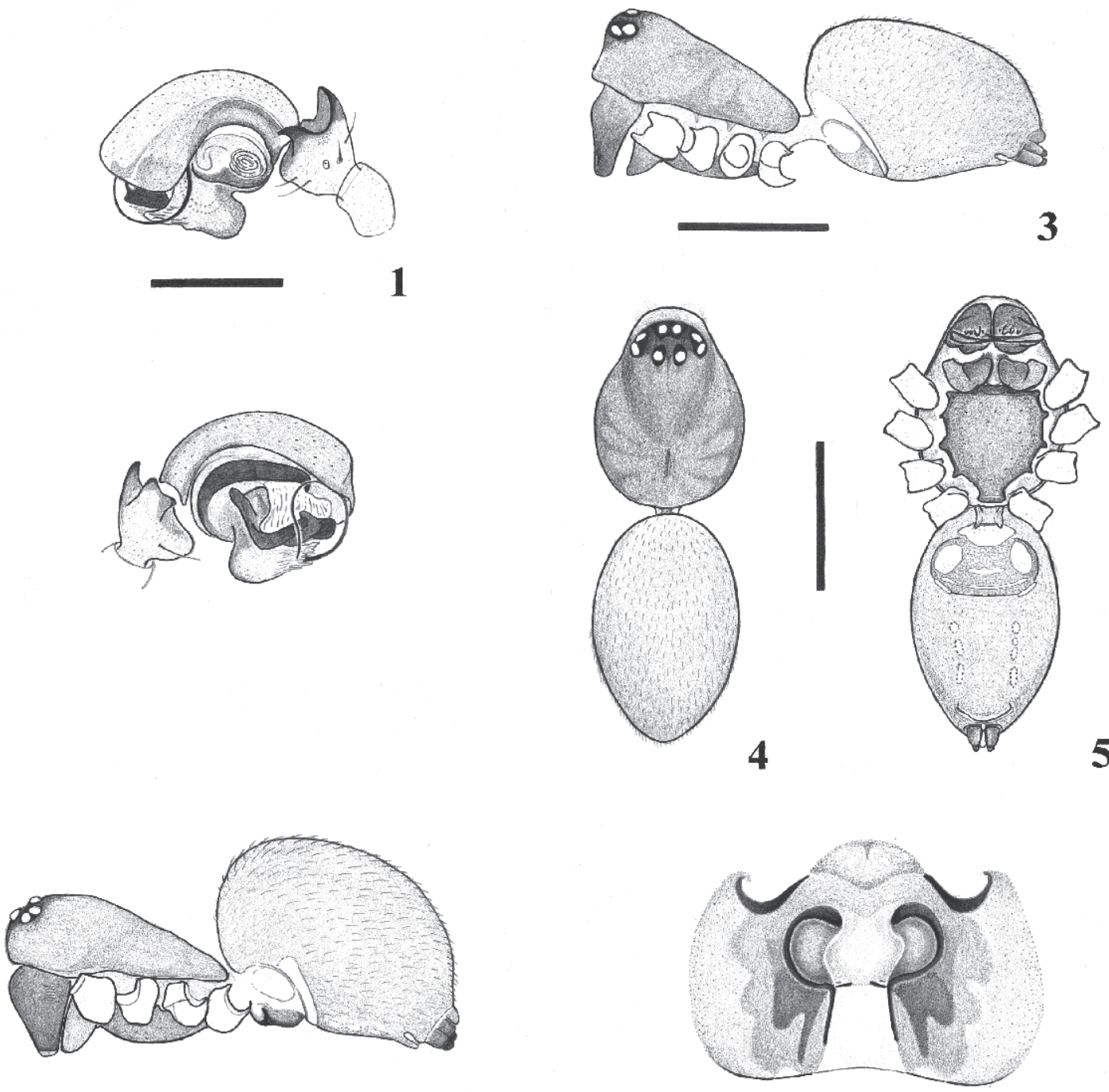

6

9
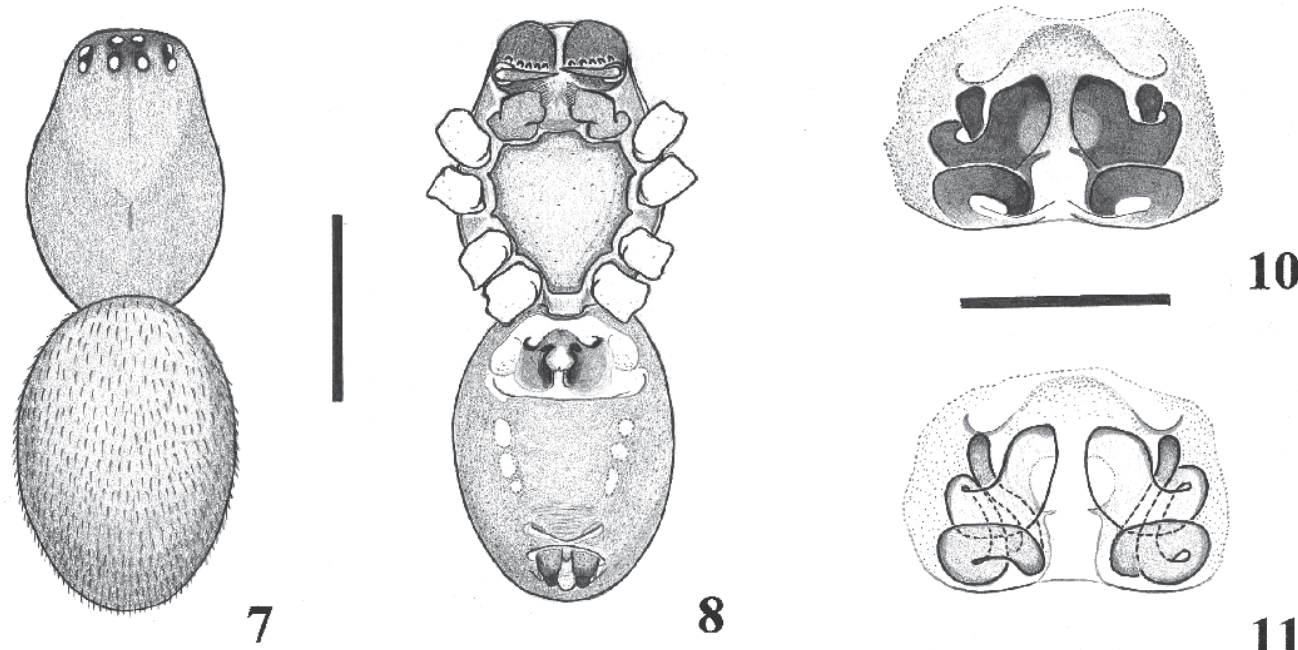

8

\section{1}

Figs. 1-11. Sphecozone tincta Millidge, 1991. 1-2, palpo do macho: 1, ectal; 2, mesal. 3-5, macho: 3, lateral; 4, dorsal; 5, ventral. 611, fêmea: 6, lateral; 7, dorsal; 8, ventral; epígino: 9, ventral; 10, dorsal; 11, dorsal clarificado. Barras: figs. 3-5, 6-8, 0,75 mm; figs. $1,2,9-11,0,25 \mathrm{~mm}$. 
largura 0,95, altura 0,97. Pernas 1,4,2,3. Comprimento I/II/ III/IV: fêmures 1,05/0,97/0,80/0,97; patelas 0,27/0,27/0,25/ 0,27 ; tíbias $0,92 / 0,85 / 0,65 / 0,87$; metatarsos $0,82 / 0,75 / 0,67 /$ 0,$82 ; \operatorname{tarsos} 0,50 / 0,47 / 0,40 / 0,44$. Total 3,56/3,31/2,77/3,37. Cálculo para a posição da tricobótria no metatarso da perna I (TmI) 0,45. Metatarsos II, III e IV com tricobótrias. Carapaça e esterno castanho-avermelhados, carapaça escurecida na área dos olhos (figs. 6, 7). Área ocular mais larga $(0,42)$ do que longa $(0,15)$. Quadrângulo ocular médio: largura anterior 0,12 , comprimento 0,15 , largura posterior 0,18. Quelíceras castanho-escuras, enditos mais claros (fig. 8). Pernas castanho-amareladas, com coxas e trocanteres amarelo-claros, patelas e tíbias castanhoescuras. Abdômen castanho-alaranjado, dorsalmente piloso (figs. 6,7); na região ventral, área das fiandeiras castanho-escura. Epígino esclerotinizado (figs. 9, 10) e com ductos sinuosos (fig. 11).

Material examinado. BRASIL, Rio Grande do Sul: Cachoeirinha (Estação Experimental do Arroz, Instituto Rio Grandense do Arroz), o', + , 20.X.2004, E. N. L. Rodrigues (MCN 38089); Capivari (Lagoa Capivari), o', 6 9, 20.V.2004, Equipe PROBIO col. (MCN 37357, 37364); Palmares do Sul (Banhado Gateados Norte),, , 07.V.2003, H. Gastal \& T. Aguzzoli col. (MCN 35710); (Banhado Gateados Sul), 3 \&, 09.V.2003, H. Gastal \& T. Aguzzoli col. (MCN 35712); (Fazenda das Almas), 3 ㅇ, 13.XI.2003, Equipe PROBIO col. (MCN 37268); (Banhado Gateados Oeste), O', 14.XI.2003, Equipe PROBIO col. (MCN 35258).

Distribuição. Sul do Brasil (Rio Grande do Sul).

\section{Sphecozone cristata Millidge, 1991}

Sphecozone cristata Millidge, 1991:173, figs. 729, 730, 735, 736, holótipo ơ, Itu, São Paulo, Brasil, 14.I.1959, A. M. Nadler col. (AMNH).

Novos registros. BRASIL. Rio Grande do Sul: São Borja (Garruchos - atualmente município), ơ, 7.XII.1975, A. A. Lise col. (MCN 3168); Bom Jesus, O', 01.IV.1988, A. B. Bonaldo col. (MCN 17388); Tainhas, O’, 02.IV.2002, E. L. C. da Silva col. (MCN 34946); Rio Grande (Estação Ecológica do Taim), ơ, 08.IV.1986, A. A. Lise col. (MCN 14986).

Distribuição. Brasil (Rio de Janeiro, São Paulo e Rio Grande do Sul).

\section{Sphecozone rostrata Millidge, 1991}

Sphecozone rostrata MiLlidge, 1991:175, figs. 743-746, holótipo $0^{7}$, Enerigilhada [grafia correta - Encruzilhada], Bahia, Brasil, XI.1973, M. Alvarenga col. (AMNH).

Novos registros. BRASIL. Rio Grande do Sul: Terra de Areia, O’, 13.XI.2001, E. L. C. da Silva col. (MCN 34947); Santa Maria (Perau Velho), o', 11.V.1998, L. Indrusiak \& Monteiro col. (MCN 33903); Santo Antônio da Patrulha (Morro Grande), 3 o", 18.VII.2002, A. B. Bonaldo col. (MCN 33107); Glorinha (São João), ơ, 14.VII.2000, A. B. Bonaldo col. (MCN 33049); Estrela Velha (Barragem de Itaúba), 4 ơ, 28.X.1999, A. L. H. Silva col. (MCN 31683); Salto do Jacuí (Horto CEEE), ơ, 26.X.1999, A. Franceschini col. (MCN 31690); Gravataí (Morro do Tigre), 10
O', 15.VII.2000, A. B. Bonaldo col. (MCN 33084); Viamão (Arroio Três Figueiras), O', 13.VII.2000, A. B. Bonaldo col. (MCN 33010); (Praia das Pombas), ơ, 15.I.2002, H. P. Romanowski col. (MCN 34865); (Parque Estadual de Itapuã), O’, IV.2002, L. E. C. Schmidt col. (MCN 34692).

Distribuição. Brasil (Bahia e Rio Grande do Sul).

\section{Sphecozone rubescens O. P.-Cambridge, 1870}

Sphecozone rubescens O. P.-CAMBRIDGE, 1870:733, pr. 44, fig. 2 , síntipos O', 2 \&, Santa Fé, Minas Gerais, Brasil, depositado no Hope Department, University Museum, Oxford (HDO); KeYSERLING, 1886: 222, pr. 19, fig. 279; SimON, 1897: 2; vaN Helsdingen, 1979: 410, figs. 8-11; Millidge, 1985: 68, figs. 260-264; Millidge, 1991: 171, figs. 726-728.

Novos registros. BRASIL. Rio Grande do Sul: Pelotas, $0^{7}$, 2 क, 10.I.2001, E. N. L. Rodrigues col. (MCN 33774).

Distribuição. Brasil (Minas Gerais, Rio de Janeiro e Rio Grande do Sul) e Argentina (Tucumán).

Agradecimentos. A Erica H. Buckup, Maria Aparecida de L. Marques e Ricardo Ott pelas sugestões. Ao pesquisador Jaime Vargas de Oliveira (IRGA) pela disponibilização da área para coleta. Ao professor Milton Mendonça Jr. (UFRGS) pela orientação no PPG-BAN que propiciou a execução desta pesquisa na EEA (IRGA). Aos revisores anônimos pelas valiosas sugestões. Ao MCN pelo uso das dependências e equipamentos.

\section{REFERÊNCIAS BIBLIOGRÁFICAS}

Helsdingen, P. J. van. 1979. Remarks on Nematogmus dentimanus Simon, with comments on the status of related genera (Araneae, Erigonidae). Bulletin of the British Arachnology Society, 4:407-413.

Keyserling, E. 1886. Die Spinnen Amerikas, Theridiidae. Nürnberg, Bauer \& Raspe. Bd. 2, Hälfte 2. 295p.

Millidge, A. F. 1980. The erigonine spiders of North America. Part 1. Introduction and taxonomic background (Araneae: Linyphiidae). The Journal of Arachnology, 8:97-107.

1985. Some linyphiid spiders from South America (Araneae, Linyphiidae). American Museum Novitates, 2836: $1-78$.

1991. Further linyphiid spiders (Araneae) from South America. Bulletin of the American Museum of Natural History, 205:1-199.

1993. A North American species of the genus Sphecozone O. P.-Cambridge, 1970 (Araneae: Linyphiidae). Bulletin of the British Arachnology Society, 9:168.

Pickard-Cambridge, O. 1870. On some new genera and species of Araneida. Proceedings of the Zoological Society of London, 1870:728-747.

Platnick, N. I. 1993. Advances in Spider Taxonomy 19881991 with Synonymies and Transfers 1940-1980. New York, New York Entomological Society, The American Museum of Natural History. 846p.

2004. The world spider catalog, version 5.0. American Museum of Natural History. Disponível em: <http:// research.amnh.org/entomology/spiders/catalog/index.html>. Acesso em: 23.11.2004.

Simon, E. 1897. Liste de arachides recueillis aux îles du Cap Vert, dans la République Argentine et le Paraguay et descriptions d'espèces nouvelles. In: Viaggio del Dott. A. Borelli nella République Argentina e nel Paraguay. Bollettino dei Musei di Zoologia e Anatomia comparata, 12(270):1-8. 